\title{
Predictive Analysis on Hypertension Treatment Using Data Mining Approach in Saudi Arabia
}

\author{
Abdullah A. Aljumah, Mohammed Gulam Ahamad, Mohammad Khubeb Siddiqui \\ College of Computer Engineering and Sciences, Salman bin Abdulaziz University, Al-Kharj, Kingdom of Saudi Arabia \\ E-mail: \{aljumah88,gulamahamad\}@hotmail.com, khubeb@ksu.edu.sa \\ Received September 11, 2011; revised October 4, 2011; accepted October 14, 2011
}

\begin{abstract}
In the present investigation, the data sets of (Non Communicable Diseases) NCD risk factors a standard report of Saudi Arabia 2005 in collaboration with World Health Organisation have been employed for regression analysis using data mining technique and that leads to the prediction of which treatment contributes more to improvement in hypertension. The Oracle Data Miner (ODM) tool has been used for the analysis of data. The data sets for different age groups in case of blood pressure treatment for hypertension for Male using different modes have been studied. The age group is in between of 15 years to 64 years. Data mining is an appropriate and sufficiently sensitive method to analyze outcomes of which mode of treatment is more effective to which age group. The Oracle data miner predicts the best mode of treatment for each group by which one can analyse the appropriate treatment. The predictions are also been compared with the help of residual plots. The residual graphs have been correlated with the predictions.
\end{abstract}

Keywords: Hypertension, Data Mining, Regression, Residual Plots, Prediction

\section{Introduction}

Data Mining is a process of discovering meaningful useful information in large data repositories. Data mining can discover valuable but hidden knowledge from databases. The applications of data mining can be found in many areas such as evaluating risks of financial investment, detection of credit card fraud, patient diagnosis etc.

Hypertension, or high blood pressure, is dangerous because it can lead to strokes, heart attacks, heart failure, or kidney disease and many more disease aliments. The goal of hypertension treatment is to lower high blood pressure and protect important organs, like the brain, heart, and kidneys from damage. Treatment for hypertension has been associated with reductions in stroke (reduced an average of $35 \%-40 \%)$, heart attack $(20 \%-25 \%)$, and heart failure (more than 50\%), according to research [1]. Hypertension is widely considered to be one of the most important risk factors for these diseases and is strongly associated with death from stroke, congestive heart failure and coronary heart disease. Using a conservative definition of essential hypertension $(>160 \mathrm{~mm} \mathrm{Hg}$ systolic or $95 \mathrm{~mm} \mathrm{Hg}$ diastolic), it is estimated that in the UK more than half of the 10 million people over the age of 65 are hypertensive. The high prevalence of the disease and ra- pidly growing number of older people suggest that hypertension is an enormous public health problem and yet detection and treatment remain relatively low [2]. All patients with blood pressure readings greater than 120/80 should be encouraged to make lifestyle modifications, such as eating a healthier diet, quitting smoking, and undergo more physical exercise. Treatment with medication is recommended to lower blood pressure to less than $140 / 90 \mathrm{~mm} \mathrm{Hg}$. For patients who have diabetes or chronic kidney disease, the recommended blood pressure is less than 130/80 mm Hg. All patients with blood pressure readings greater than $120 / 80$ should be encouraged to make lifestyle modifications, such as eating a healthier diet, quitting smoking, and undergo more physical exercise. Treatment with medication is recommended to lower blood pressure to less than $140 / 90 \mathrm{~mm} \mathrm{Hg}$. For patients who have diabetes or chronic kidney disease, the recommended blood pressure is less than 130/80 $\mathrm{mm} \mathrm{Hg}$.

Hypertension is a major risk factor for stroke and coronary heart disease, and is a major contributor to the onset and progression of chronic heart failure and chronic kidney failure. High blood pressure is often called the "silent killer" because it has no symptoms and can go undetected for years. Patients with hypertension should routinely provide advice on smoking, nutrition, alcohol use, 
physical activity and body weight.

Modes of Treatment: As mentioned in World Health Organisation's NCD report of Saudi Arabia following five types of treatments that are discussed below:

1.1. Drug.

1.2. Diet.

1.3. Weight Reduction.

1.4. Smoke Cessation.

1.5. Exercise.

\subsection{Drug}

Diuretics are usually recommended as the first line of therapy for most people who have high blood pressure. If one drug doesn't work or is disagreeable, other types of diuretics are available.

However, your doctor may start a medicine other than a diuretic as the first line of therapy if you have certain medical problems. For example, ACE inhibitors are often a good choice for a people with diabetes. If your blood pressure is more than $20 / 10 \mathrm{mmHg}$ higher than it should be, your doctor may consider starting you on two drugs.

\subsection{Diet}

A variety of dietary modifications are beneficial in the treatment of hypertension, including reduction of sodium intake, moderation of alcohol, weight loss in the obese, and possibly increasing potassium and calcium intake, and ingestion of a vegetarian diet or fish oil supplements [3] Potassium supplements ( 2 - 4 grams daily) have been shown to moderately decrease blood pressure. Fruits and vegetables are excellent sources of potassium. Foods high in omega-3 fatty acids have positive effects on hypertension and cardiovascular disease by relaxing arteries and thinning the blood. Dietary recommendations suggest avoiding too much sodium. Excessive sodium intake is linked with high blood pressure or hypertension in some people. The suggested range is 1100 to $3300 \mathrm{mg}$ per day.

\subsection{Weight}

There is a direct association between blood pressure and body weight and/or abdominal adiposity. Weight loss studies show that clinically significant blood pressure reductions can be achieved by modest weight loss in people with and without hypertension and that blood pressure reduction is proportional to weight loss. [4] Every $1 \%$ reduction in body weight lowers systolic blood pressure by an average of $1 \mathrm{mmHg}$ and weight reduction confers a range of other cardiovascular health benefits including reduced insulin resistance and hyperlipidaemia, and reduced risk of left ventricular hypertrophy and obstructive sleep apnoea. [5] states that losing $4.5 \mathrm{~kg}$ reduces blood pressure or prevents hypertension in a large proportion of overweight people, while losing $10 \mathrm{~kg}$ can reduce systolic blood pressure by $6-10 \mathrm{mmHg}$. In overweight patients with hypertension, weight-reducing diets can achieve a $3-9 \%$ decrease in body weight and may reduce systolic and diastolic blood pressure by approximately $3 \mathrm{mmHg}$.

\subsection{Smoke Cessation}

Smoking is a strong independent risk factor for cardiovascular disease. Quitting is acknowledged to be one of the most effective lifestyle interventions for preventing cardiovascular disease and premature deaths. Smoking causes an immediate increase in blood pressure and heart rate that persists for more than 15 minutes after one cigarette. People who smoke show higher ambulatory blood pressure levels than non-smokers, although smoking is known to increase the risk of developing hypertension, there is currently no evidence that smoking cessation directly reduces blood pressure in people with hypertension [4]. Elevated blood pressure and smoking are the two most important risk factors for subarachnoid haemorrhage in the Asia-Pacific region. The risk of myocardial infarction is 2-6 times higher and the risk of stroke is three times higher in people who smoke, compared with non-smokers [5].

\subsection{Exercise}

It is clear that exercise (physical activity) lowers resting and daytime ambulatory blood pressure. In clinical trials of people with hypertension, regular aerobic activity reduced systolic blood pressure by an average of 6.9 $\mathrm{mmHg}$ and diastolic blood pressure by $4.9 \mathrm{mmHg}$. [6] Regular exercise has an independent cardio protective effect. Regular exercise is associated with an increase in high-density lipoprotein cholesterol and with reductions in body weight, waist circumference, percentage body fat, insulin resistance, systemic vascular resistance, and plasma noradrenalin and plasma rennin activity [1]. Both aerobic and resistance training have been shown to facilitate anti-hypertensive responses, although aerobic exercise has been more largely studied. Specifics concerning optimal intensity and length of the exercise program are yet to be fully determined, however, moderate intensity exercise performed for at least 30 minutes on most days of the week still remains to be the 'minimal' yet effective recommendation necessary for prevention and treatment of hypertension, as well as for promoting overall health. Warm up for no less than $5-10$ minutes to ensure an 
appropriate preparation for the cardiovascular system. Emphasize non weight-bearing activities, as most hypertensive is obese or elderly. Duration of 20 - 30 minutes of exercise is recommended.

\section{Related Works}

The literature and survey reveals many results on hypertension treatment, As per the university of York report [2], hypertension is widely considered to be one of the most important risk factors for these diseases and is strongly associated with death from stroke, congestive heart failure and coronary heart disease. It is estimated that in the UK more than half of the 10 million people over the age of 65 are hypertensive. The high prevalence of the disease and rapidly growing number of older people suggest that hypertension is an enormous public health problem and yet detection and treatment remain relatively low [3]. they have determined the prevalence of hypertension among Saudis in both genders, between the ages of 30 - 70 years in rural as well as urban communities. They have carried out the study on 17,230 subjects. This work is part of a major national study on Coronary Artery Disease in Saudis Study (CADISS). This is a community-based study conducted by examining subjects in the age group of 30 - 70 years of selected households during a 5 year period between 1995 and 2000 in Saudi Arabia. Data has been obtained from history using a validated questionnaire, and examination including measurement of blood pressure. The data were analyzed to provide prevalence of hypertension. In this work the Logistic regression technique is used to develop a risk assessment model for prevalence of hypertension. The prevalence of hypertension was $26.1 \%$ in crude terms. Increasing weight showed significant increase in prevalence of hypertension in a linear relationship. [6] Screened 13,700 individuals of both sexes in all age groups. Applying the criteria of W.H.O. of blood pressure $>160 / 95 \mathrm{mmHg}$ as hypertension, they found prevalence of $9.1 \%$ and $8.7 \%$ systolic and diastolic hypertension, respectively. Among the adults ( $>18$ years), 5.3\% had systolic hypertension, while $7.9 \%$ had diastolic hypertension. The majority $(>75 \%)$ of those with hypertension were 40 years of age. In the age group 40 - 75 years, females had a higher prevalence $(15.7 \%)$ of systolic hypertension compared to males $(\mathrm{p}<0.05)$, while males had a higher prevalence $(8.2 \%)$ of diastolic hypertension compared to their female counterparts $(6.6 \%)(\mathrm{p}<0.001)$. [7] developed the predictive analysis to classify sixteen diseases based on discharge summary in this study, the authors have predicted whether each disease is present, absent or questionable. This has been a multiclass classification task they have evaluated these sixteen diseases and showed that it improves significantly over voting and stacking, when they had used for multiple class classification. [8] have presented the hypertension studies in Saudi Arabia compared a few isolated and three comprehensive studies covering the whole Kingdom of Saudi Arabia and show that different investigators found different prevalence of hypertension in different areas of the kingdom, they unify the diagnostic procedures and to determine the factors behind such significant differences. [9] Examined individuals with ages ranging between 18 50 years in the northern province of Saudi Arabia. He reported that if diastolic blood pressure of $>90 \mathrm{mmHg}$ was considered as the cut-off point for definition of hypertension, the prevalence of hypertension would be $15.2 \%$, while if $>95 \mathrm{~mm} \mathrm{Hg}$ was used, the prevalence of hypertension would be 5.25\%. [10] Reported results of a National Nutrition Survey in which blood pressure was checked in 17,892 individuals from age 12 years and above. There were 6260 adults over 18 years of age. The prevalence of systolic and diastolic hypertension was determined by using two cut-off values i.e. $>160 / 95$ and $>140 / 90$. The prevalence of systolic and diastolic hypertension in the adult population was $5.3 \%$ and $7.3 \%$, respectively, using the cut-off value of 160/95. There were significant geographical variations. The prevalence of systolic blood pressure was the highest in Taif, Farasan and Hail and lowest in Asir, Jizan and AlMadina. The prevalence of diastolic blood pressure was highest in Al-Qassim, Jeddah, Tabouk and Hail and lowest in Makkah. Interestingly, females generally showed a higher prevalence compared to the male counterparts in all geographical areas.

\section{Material and Methods}

\subsection{Data Collection}

The data has been is collected from World Health Organisation's Data and Statistics, the data is NCD risk factor, standard report of Ministry of Health, Saudi Arabia, 2005 [11].

Five tables have been designed including drug, diet, weight, smoke_cession and exercise. Each table indicates the mode of hypertension treatment on male patients.

Each table includes 6 columns (sr, age, N, small_n, Percentage, se), "SR" indicates the serial number, which acts as a unique identifier in the table (primary key). "Age" indicates the age of patients. " $\mathrm{N}$ " indicates the total number of patient of each age group e.g. in Table 2 ages 15 - 24 has 10 patients. "small_n" column shows the number of patients who have been cured with the particular type of treatment. The percentage indicates the percent of cured patients by specific mode of treatment. Column "se" indicates the standard error. Tables are indicated below: (Tables 1-5). 
Table 1. Drug.

\begin{tabular}{cccccc}
\hline SR_NO & AGE & N & SMALL_N & PERCENTAGE & SE \\
\hline 1 & $15-24$ & 10 & 3 & 32.2 & 16.2 \\
2 & $25-34$ & 19 & 6 & 29.9 & 11.5 \\
3 & $35-44$ & 35 & 23 & 64.3 & 8.8 \\
4 & $45-54$ & 77 & 62 & 81.3 & 4.8 \\
5 & $55-64$ & 99 & $90 \mathrm{~s}$ & 90.8 & 2.6 \\
\hline
\end{tabular}

Table 2. Diet.

\begin{tabular}{cccccc}
\hline SR_NO & AGE & N & SMALL_N & PERCENTAGE & SE \\
\hline 1 & $15-24$ & 10 & 2 & 19.7 & 13 \\
2 & $25-34$ & 19 & 2 & 10.8 & 6.8 \\
3 & $35-44$ & 35 & 21 & 60.4 & 9.5 \\
4 & $45-54$ & 77 & 45 & 58.7 & 6.6 \\
5 & $55-64$ & 99 & 52 & 53 & 8.5 \\
\hline
\end{tabular}

Table 3. Weight.

\begin{tabular}{cccccc}
\hline \multirow{2}{*}{ SR_NO } & AGE & N & SMALL_N & PERCENTAGE & SE \\
\hline 1 & $15-24$ & 10 & 2 & 19.7 & 13 \\
2 & $25-34$ & 19 & 5 & 27 & 10.4 \\
3 & $35-44$ & 35 & 17 & 48.5 & 9.1 \\
4 & $45-54$ & 77 & 26 & 33.4 & 5.3 \\
5 & $55-64$ & 99 & 39 & 39.7 & 7.8 \\
\hline
\end{tabular}

Table 4. Smoke cessions.

\begin{tabular}{cccccc}
\hline SR_NO & AGE & N & SMALL_N & PERCENTAGE & SE \\
\hline 1 & $15-24$ & 10 & 3 & 32.3 & 16.2 \\
2 & $25-34$ & 19 & 2 & 10.8 & 7.3 \\
3 & $35-44$ & 35 & 4 & 11.7 & 5.5 \\
4 & $45-54$ & 77 & 13 & 16.7 & 4.4 \\
5 & $55-64$ & 99 & 32 & 13.3 & 2.5 \\
\hline
\end{tabular}

Table 5. Exercise.

\begin{tabular}{cccccc}
\hline SR_NO & AGE & N & SMALL_N & PERCENTAGE & SE \\
\hline 1 & $15-24$ & 10 & 3 & 32.3 & 16.2 \\
2 & $25-34$ & 19 & 6 & 33.3 & 10.2 \\
3 & $35-44$ & 35 & 13 & 36.9 & 7.9 \\
4 & $45-54$ & 77 & 23 & 29.9 & 5.6 \\
5 & $55-64$ & 99 & 28 & 27.9 & 5.4 \\
\hline
\end{tabular}

\subsection{Tools and Techniques}

We use the oracle data miner version 10.2.0.3.0.1, build 2007 for the mining activity, that act as a client and ora- cle $10 \mathrm{~g}$ database release 10.2.0.3.0 as a server as mention in Figure 1, whole database for hypertension is designed in oracle $10 \mathrm{~g}$ database. The database include five base table that are drug, diet, weight, smoke cession and exercise each table include six columns.

We use the oracle data miner version 10.2.0.3.0.1, build 2007 for the mining activity, that act as a client and oracle $10 \mathrm{~g}$ database release 10.2.0.3.0 as a server as mention in Figure 1, whole database for hypertension is designed in oracle $10 \mathrm{~g}$ database. The database include five base table that are drug, diet, weight, smoke cession and exercise each table include six columns.

The first tier of the above Figure 1 is the database tier where data and metadata is prepared and stored, the second tier is called Data Mining Application where the algorithms process the data and store the results in the database and the third tier is the client or Front-End layer, which facilitates the parameter settings for Data Mining Application and visualization of the results in interpretable form.

Oracle Data Miner is graphical user interface for Oracle Data Mining (Release 10.1) that helps data analysts mine their Oracle data to find valuable hidden information, patterns, and new insights. Data analysts can mine data with Oracle Data Miner's easy-to-use wizards that guide them through the data preparation, data mining, model evaluation, and model scoring process. As the data analyst transforms the data, builds models, and interprets results.

Oracle Data Miner can automatically generate code needed to transform the data mining steps into an integrated data mining.

ODM [12] is applicable in a variety of business, public sector, health care, and other environments.

We adopt Oracle Data Miner (ODM) for analysis and predicting the data, we design the database in Oracle $10 \mathrm{~g}$ that act as server and ODM act as a client.

For running the Oracle Data Miner following privileges

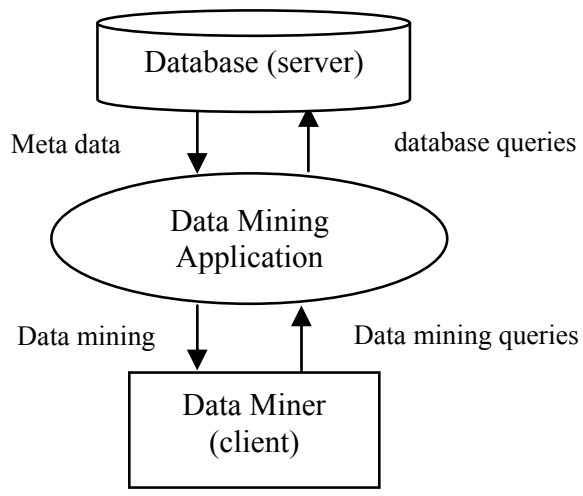

Figure 1. Shows the three tier architecture flow of data and queries between clients (ODM) to server (database). 
are required to that schema which it would connects to oracle database.

We had applied the regression technique which is well known statistics technique that the data mining commonly utilize.

There are five basic tables in database. Each table shows the mode of hypertension treatment i.e. drug, diet, weight, smoke cession, exercise.

In our case it takes "percentage" as numerical attribute which acts as its target for prediction of the results.

\subsection{Regression}

Regression is a data mining function that predicts a number. The analysis used to model the relationship between one or more independent or predictor values and dependent or response variable. In the present context of data mining the predictor variables or attributes of interest describing the tuple which are known values. The response variable is what we want to predict.

A regression task begins with a data set in which the target values are known. In the present investigation the data set values of 'percentage' of patients treated in each group and in each mode of treatment.

Residual Plots: A residual plots is scatter plot where the $\mathrm{x}$-axis is the predicted value of $\mathrm{x}$, and the $\mathrm{y}$-axis is the residual for $\mathrm{x}$. The residual is the difference between the actual value and the predicted value of $\mathrm{x}$.

\section{Experimental Analyses}

We have carried out the experimental analysis on the NCD data of Saudi Arabia using Oracle Data Miner tool. For prediction of data, the five age groups are classified into two age groups Young and Old. Young group is denoted as "Y" and Old age group is denoted as "O". The prediction is denoted as " $p$ ". The young group includes the age group of (15 - 24, 25 - 34 and 35 - 44) and old group includes the age group of (35 - 44, 45 - 54 and 55 - 64) in both young and old age group includes the "35 - 44" as common age group. It is a upper limit for young group and lower limit for old group. The equation that used to calculate the prediction of both young and old age groups is given below.

$$
\begin{aligned}
& \mathrm{p}(\mathrm{Y})=[\mathrm{p}(1)+\mathrm{p}(2)+\mathrm{p}(3)] \\
& \mathrm{p}(\mathrm{O})=[\mathrm{p}(3)+\mathrm{p}(4)+\mathrm{p}(5)]
\end{aligned}
$$

where 1, 2, 3, 4 and 5 are the serial numbers of table where 1 indicates the age " $15-24$ ", 2 indicates the age " 25 - 34", 3 indicates the " 35 - 44", 4 indicates the "45 54 " and 5 indicates the " 55 - 64".

The results of prediction based on types of treatment of hypertension disease are as give below:

\subsection{Drug}

Above Figure 2 is an evidence to show that the drug treatment of hypertension in older age people saves lives and prevents the unnecessary morbidity.

$$
\begin{aligned}
& \mathrm{p}(\mathrm{Y})=((-18.608)+(-41.007)+8.9999)=-50.616 \\
& \mathrm{p}(\mathrm{O})=(8.999+15.8845+(-14.782))=10.1015 \\
& \mathrm{p}(\mathrm{O})>\mathrm{p}(\mathrm{Y})
\end{aligned}
$$

The above prediction clearly states that drug is more effective to older age people. Hence, this treatment in older people with hypertension can continue to contribute the effective treatment of hypertension. As per Medical Research Council studies, in which thiazide diuretics were associated with a greater than $40 \%$ reduction in the risk of stroke in patients with isolated systolic hypertension [13]. also showed the differences in tolerability between agents, and the particular difficulty in controlling blood pressure to target values especially with angiotensin converting enzyme inhibitors in black patients.

\subsection{Diet}

The predictions mentioned in Figure $\mathbf{3}$ are comparable to group of both the younger and older age.

$$
\begin{aligned}
& \mathrm{p}(\mathrm{Y})=(8.8287+8.7335+18.9181)=36.4803 \\
& \mathrm{p}(\mathrm{O})=(18.9181+29.1851+17.7022)=65.8054 \\
& \mathrm{p}(\mathrm{O})>\mathrm{p}(\mathrm{Y})
\end{aligned}
$$

Diet control is more effective to old age people because value of " $p$ " in old age people is greater than young age people, as predictive analysis as stated in Figure 2. All the predictions for all age groups are positive, and none of them is negative, which clearly states that diet treatment for hypertension is effective to all age group people. Hypertension can lead to other health complications such as strokes, kidney failure, impaired vision, heart attack, and heart failure. People with a low calcium intake seem

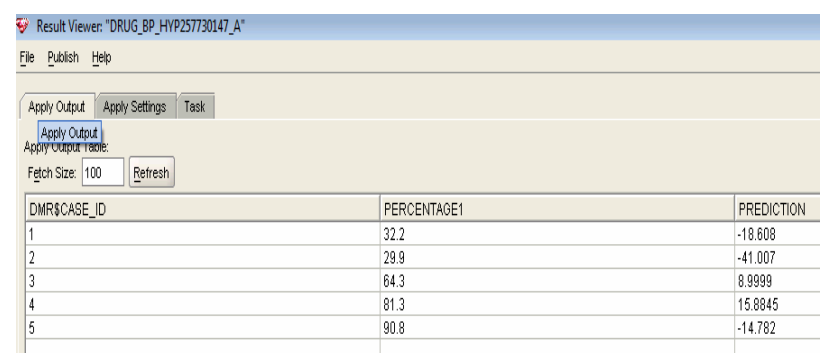

Figure 2. States the prediction of treatment by drug. 


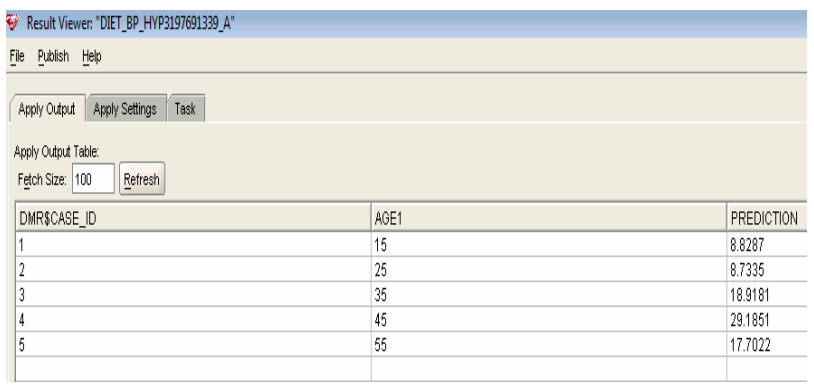

Figure 3. States the prediction of treatment by diet.

to be at increased risk for hypertension. Everyone should meet the Dietary Reference Intake (DRI) for calcium every day. As per our predictive analysis the $\mathrm{p}(\mathrm{O})>\mathrm{p}$ (Y) therefore the intake of calcium for adults $1000 \mathrm{mg}$ per day and for older people, $1200 \mathrm{mg}$ is recommended. Potassium has an important role in blood pressure treatment. People trying to control hypertension often are advised to decrease sodium, increase potassium, watch their calories, and maintain a reasonable weight.

\subsection{Weight}

As per the above prediction Figure 4 for weight reducetion, it is not an important factor for young age people, where as this is an important factor for the older age people.

$$
\begin{aligned}
& \mathrm{p}(\mathrm{Y})=(9.8712+(-2.0424)+24.3366)=32.1654 \\
& \mathrm{p}(\mathrm{O})=(24.3366+25.0085+11.6748)=61.0199 \\
& \mathrm{p}(\mathrm{O})>\mathrm{p}(\mathrm{Y})
\end{aligned}
$$

With the increase in the weight obesity is a one of the risk factor for hypertension and other cardiovascular diseases. As Body Mass Index (BMI) increases, so does the risk of hypertension. It is important to assess BMI and waist circumference in each individual. Using BMI, patients can be classified as normal weight (BMI 18.5 $\left.24.9 \mathrm{~kg} / \mathrm{m}^{2}\right)$, overweight $\left(25-29.9 \mathrm{~kg} / \mathrm{m}^{2}\right)$ or obese $(\geq 30$ $\mathrm{kg} / \mathrm{m}^{2}$ ) (Ministry of Health, Saudi Arabia, Saudi Hypertension Management Guidelines, 2007). For those obese patients a weight management plan should be con-

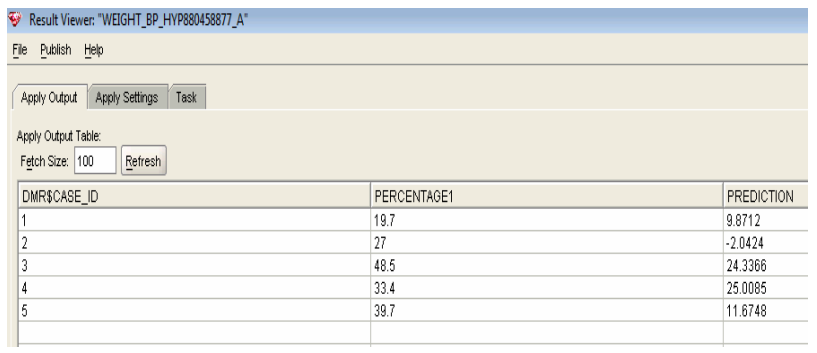

Figure 4. States the prediction of treatment by weight. structed and discussed with the patient. Options available include lifestyle modification (including behaviour therapy), pharmacotherapy, and bariatric surgery.

People who are overweight are more likely to have highnormal to mild high blood pressure. Studies revealed that about one-third of patients with high blood pressure are overweight. Even moderately obese adults have double the risk of hypertension than people with normal weights. In fact, the increase in blood pressure in aging may be due to weight gain. Children and adolescents who are obese are at greater risk for high blood pressure when they reach adulthood. Statistics show that most people who have high blood pressure are also overweight. If a person is overweight or has gained weight over time, he is advised to cut down on calories and lose weight.

The above calculation states that there is a wide difference almost twice between the predictions of young age people and older age people. So it is concluded that older age people have to be more concentrate on weight reduction treatment of hypertension.

\subsection{Smoke Cession}

It is predicted in the above table that older people is recommended to stop smoking which is predicted in the above Figure 5. The young may not be so affected when compared to old aged people.

$$
\begin{aligned}
& \mathrm{p}(\mathrm{Y})=(4.2379+3.5432+5.2072)=12.9883 \\
& \mathrm{p}(\mathrm{O})=(5.2072+8.3329+4.5814)=18.1215 \\
& \mathrm{p}(\mathrm{O})>\mathrm{p}(\mathrm{Y})
\end{aligned}
$$

The above result indicates that smoking is more dangerous to old age people in comparison to young age people. However, it is concluded that to avoid smoking habit is better option for older age group people as per the above results. Smoking is a strong independent risk factor for hypertension and also for cardiovascular disease. Smoking cessation markedly reduces overall cardiovascular risk, including the risk of coronary heart

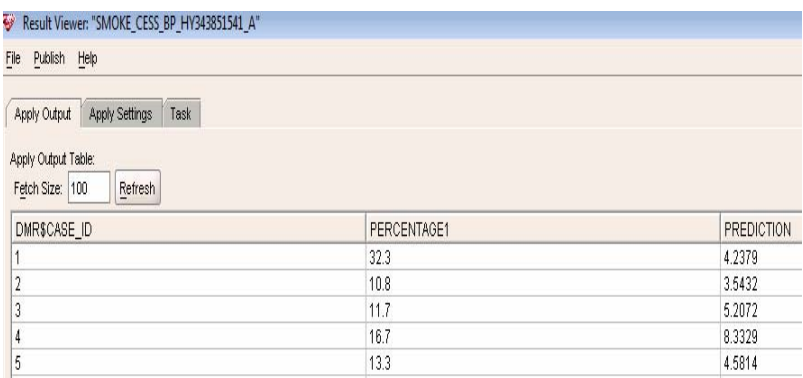

Figure 5. States the prediction of treatment by smoke cession. 
disease and stroke, compared with continued smoking. In patients with coronary heart disease, smoking cessation is associated with a $36 \%$ reduction in the risk of all-cause mortality [2]. Although smoking is known to increase the risk of developing hypertension. All patients are unambiguous advice to stop smoking, assess for nicotine dependence (e.g. time of last cigarette, withdrawal symptoms) and offer counselling. Quitting is acknowledged to be one of the most effective lifestyle interventions for preventing hypertension, cardiovascular disease and premature deaths.

\subsection{Exercise}

The Figure 6 above shows the prediction of exercise mode of treatment to hypertension patient of Saudi Arabia. It shows the exercise is effective to both young and old age peoples.

$$
\begin{aligned}
& \mathrm{p}(\mathrm{Y})=(14.4113+19.0749+15.0142)=48.5004 \\
& \mathrm{p}(\mathrm{O})=(15.0142+19.0749+14.9583)=49.0474 \\
& \mathrm{p}(\mathrm{Y})=\mathrm{p}(\mathrm{O})\{\text { approximately equal }\}
\end{aligned}
$$

As per above there is no difference in the prediction of young age people and old age people. So, exercise mode of treatment is effective to both the groups.

Exercise reduces blood pressure in hypertensive by 5 to 10 points - both systolic and diastolic. The encouragement of regular exercise is not only useful as a treatment method for individuals with hypertension, but should also be advocated as a means for prevention.

The beneficial effect of regular exercise in hypertension is not limited to reduction of blood pressure only, but also the physical activities (e.g. recreational sports, skiing, gymnastics, heavy gardening, hunting, fishing and walking/jogging) can reduce the risk of hypertension in all age groups. Thus the physical activity and regular exercise can protect against hypertension.

Residual Plots: The below figures states the residual plot of each type of hypertension treatment, each treat-

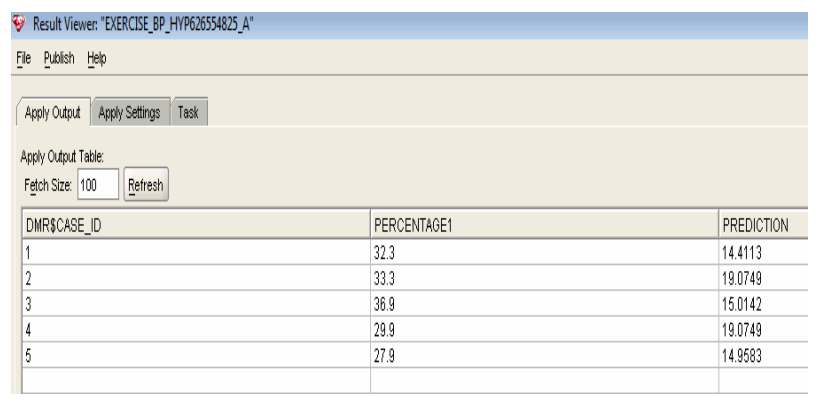

Figure 6. States the prediction of treatment by exercise. ment holds two plots first is actual residual plot and second is predicted residual graphs (Figures 7-11).

\section{Discussions}

The data presented in this study report have overall higher prevalence of hypertension among adult Saudi male population. Clearly, hypertension is a major risk factor affecting large portion of the Saudi community and make them vulnerable of acquiring cardiovascular diseases (CVD), peripheral vascular disease PVD, as well as renal and cerebrovascular diseases. As hypertension is associated with an increase in risk for cardiovascular disease, it is vital that effective interventions are advocated to reduce overall morbidity and mortality. Further analysis of our
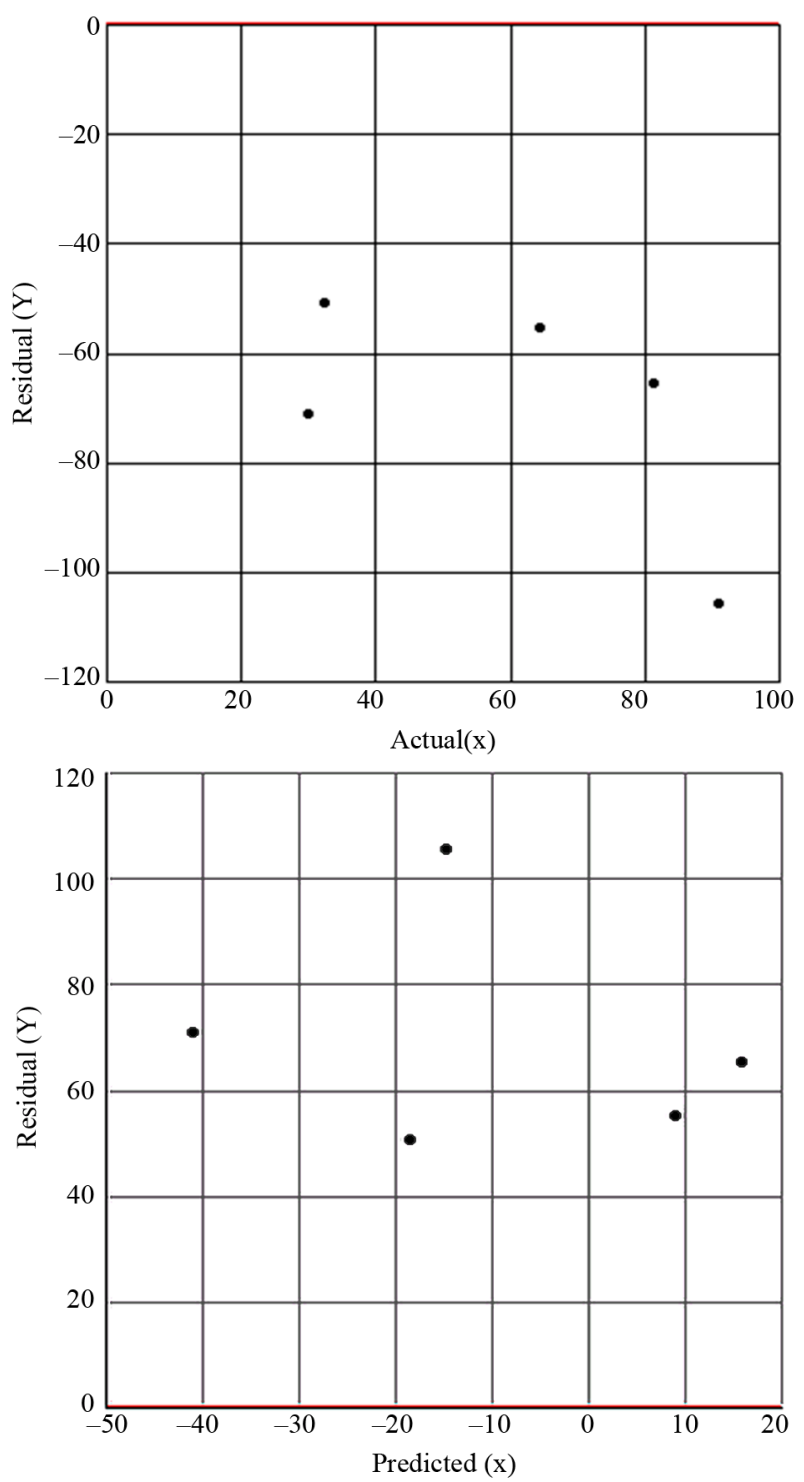

Figure 7. Residual plot for drug treatment. 

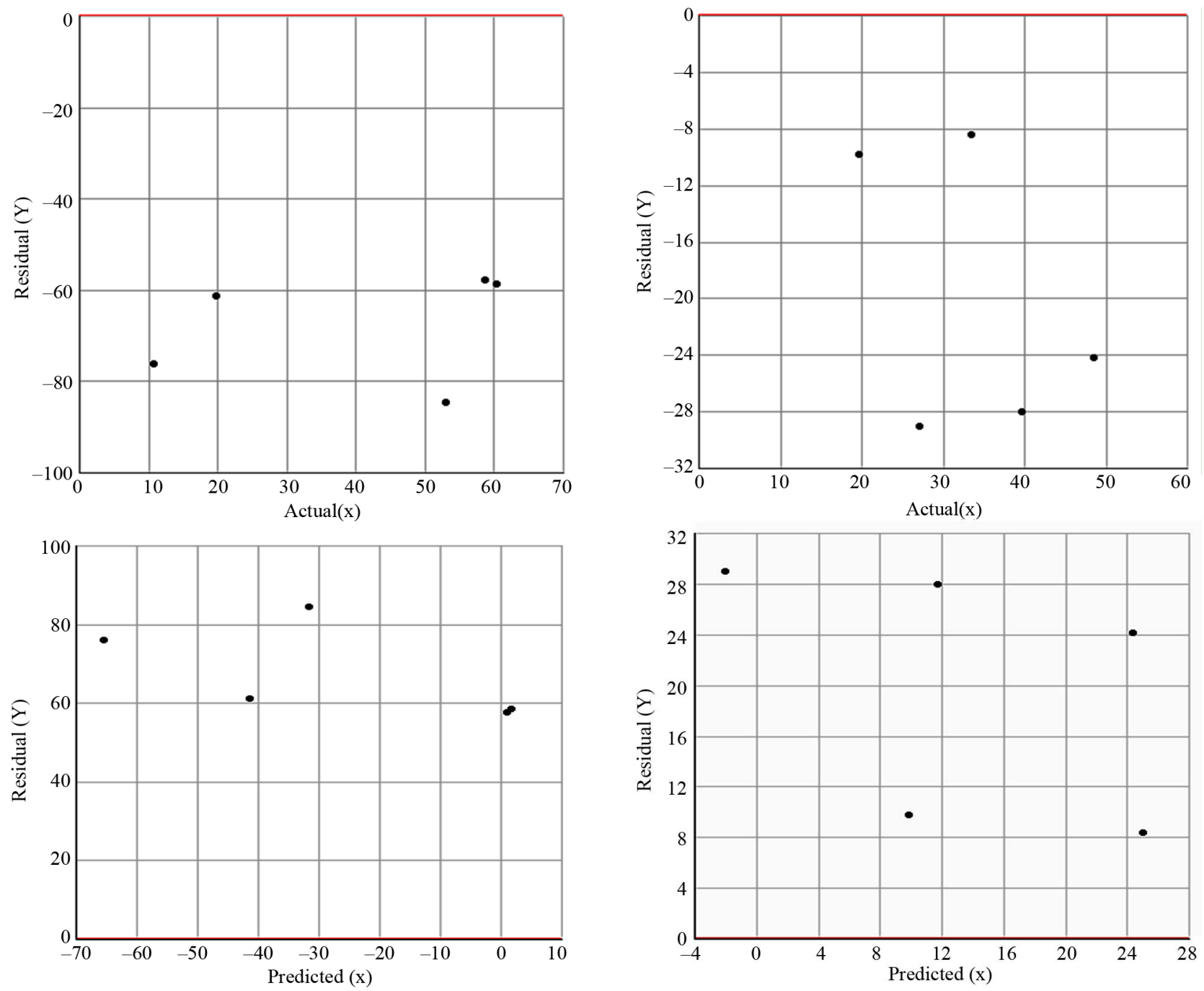

Figure 8. Residual plot for diet treatment.

Figure 9. Residual plot for weight treatment.
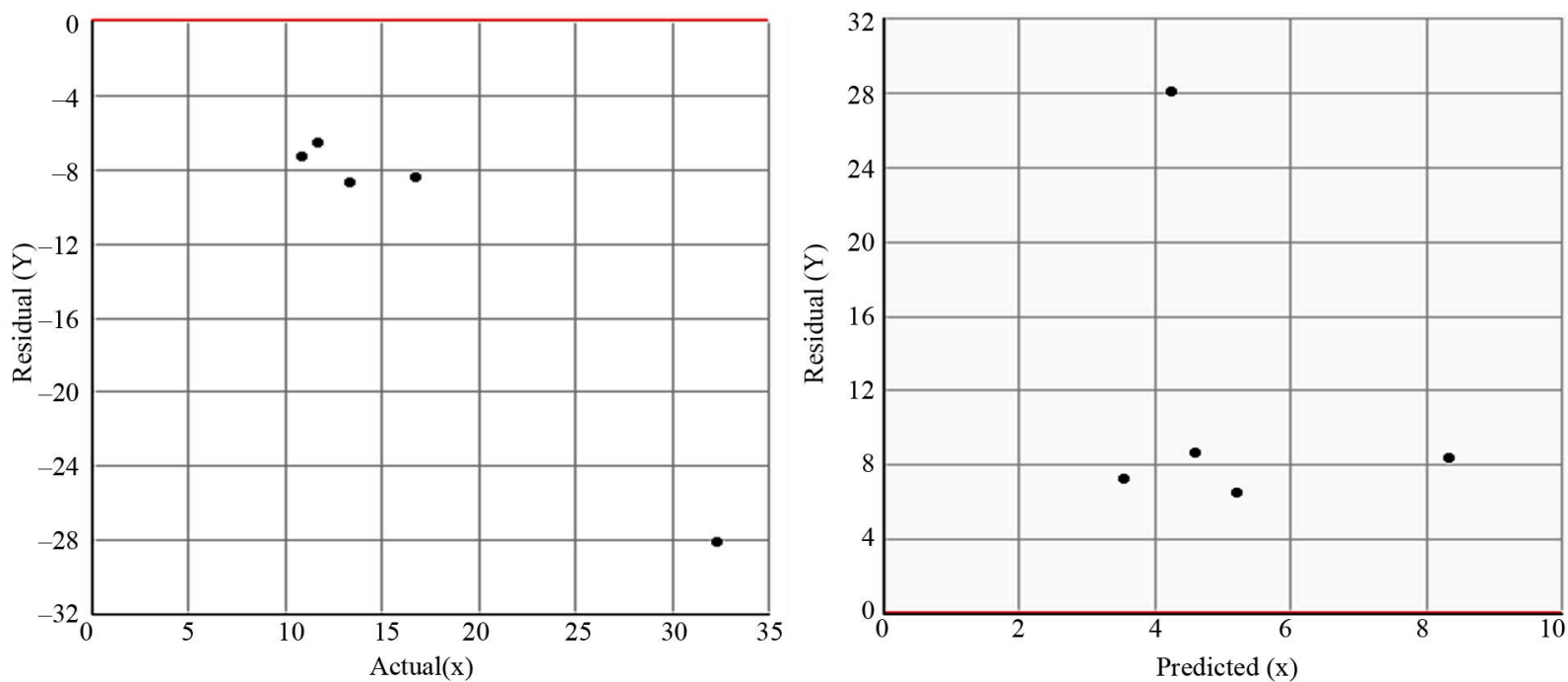

Figure 10. Residual plot for smoke cessation 

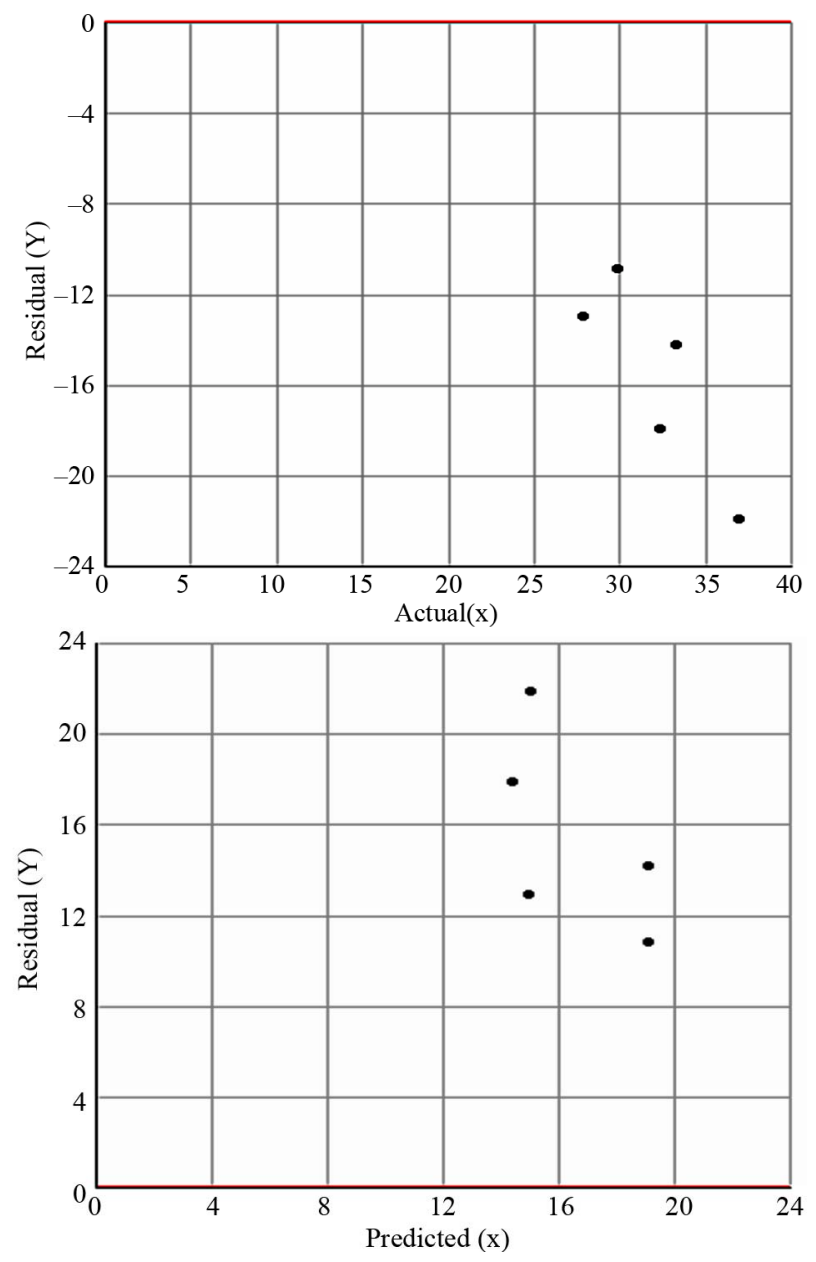

Figure 11. Residual plot for exercise treatment.

data in the present study, clearly demonstrate an increaseing prevalence of hypertension with increasing age and this increase is due to mainly increase in systolic hypertension. We also found that the all the five modes of treatments are effective for older people because prevalence of hypertension at older ages is higher in men.

A large number of the hypertensive patients $(66.9 \%)$ are unaware of having hypertension which is the silent killer. High blood pressure can occur in children or adults, but it's more common among people over age 35. It's particularly prevalent in elderly people, obese people, heavy drinkers and women who are taking birth control pills. People with diabetes mellitus, gout or kidney disease are more likely to have high blood pressure, too.

The depicted residual plots obtained from the regression results. The residual plots of each type treatments having two categories first one is actual residual plot and second is predicted residual plot. Actual residual plot shows the treatment are below zero axis indicating the negative response on other hand the predicted residual plots when compared to actual residual plot the factors are above zero showing that the predictions are positive and appropriate. This confirms the less error in the predictions of each treatment.

The residual plots are almost random in nature so which ascertain the fact that the regression analysis is non linear and Euclidian in nature.

\section{Conclusions}

The study concludes that hypertension is increasing prevalence in Saudi Arabia affecting more than one fourth of the adult Saudi population. We have recommended aggressive management of hypertension as well as screening of male adults for hypertension early to prevent its damaging consequences if left untreated. Public health awareness of simple measures, such as low salt diet, exercise, and avoiding obesity, to maintain normal arterial blood pressure need to be implemented by health care providers. The above results in section 4 in all five modes of treatment the $\mathrm{p}(\mathrm{O})>\mathrm{p}(\mathrm{Y})$ it is found that hypertension cases more in older age people and it is obvious that hypertension is a prevalent risk factor in Saudi Arabia. All five modes of treatments are actively employed in treating older people. All five modes of treatments are actively employed in treating older people. That needs intervention to be controlled. This may include dietary measures as well as lifestyle modifications, particularly, drug, diet, weight reduction, smoke cession and exercise. Each type of treatment have highest prediction rate in older age people.

\section{Acknowledgements}

We would like to give acknowledge and thanks to Salman bin Abdulaziz University for the support while completing this research work and to all my colleagues who have taken the time to review the paper and comment on this paper prior to publication. Special thanks to our friends, who have been unselfishly extending their efforts and understanding.

To our parents who have always been very understanding and supportive. And above all, to the Almighty Allah, who never cease in loving us and for the continued guidance and protection.

\section{References}

[1] M. M. Al-Nozha, M. S. Ali and A. K. Osman, "Arterial hypertension in Saudi Arabia," Annals of Saudi Medicine, Vol. 17, No. 2, 1997, pp. 170-174.

[2] "Effective Matters," Vol. 4, No. 2, 1999, The University of York. http://www.york.ac.uk/inst/crd/EM/em42.pdf 
[3] M. M. M. Al-Nozha, A. Moheeb, A. R. Mohammed, K. Z. Mohamed, N. B. Khan, Y. Y. Al-Mazrou, M. A. Al-Maatouq, K. Al-Marzouki, A. Al-Khadra, M. S. Nouh, S. S. Al-Harthi, M. S. Al-Shahid and A. Al-Mobeireek, "Hypertension in Saudi Arabia," Saudi Medical Journal, Vol. 28, No. 1, 2007, pp. 77-84.

[4] G. Mancia, G. De Backer, A. Dominiczak, R. Cifkova, R. Fagard, G. Germano, et al., "Guidelines for the Management of Arterial Hypertension, the Task Force for the Management of Arterial Hypertension of the European Society of Hypertension (ESH) and of the European Society of Cardiology (ESC)," Journal of Hypertension, Vol. 25, 2007, pp. 1105-1187. doi:10.1097/HJH.0b013e3281fc975a

[5] National Heart Foundation of Australia. Guide to management of hypertension 2008. Assessing and Managing Raised Blood Pressure in Adults. NHF; 2008.

http://www.heartfoundation.org.au/Professional_Informat ion/Clinical_Practice/Hypertension.htm

[6] R. H. Fagard and V. A. Cornelissen, "Effect of Exercise on Blood Pressure Control in Hypertensive Patients," European Journal of Cardiovascular Prevention and Rehabilitation, Vol. 14, 2007, pp. 12-17. doi:10.1097/HJR.0b013e3280128bbb

[7] I. Goldstein and O. Uzunor, "Specializing for Predicting
Obesity and its Co-Morbidites," Journal of Biomedical Informatics, Vol. 42, 2009, pp. 873-886. doi:10.1016/i.jbi.2008.11.001

[8] M. A. El-Hazmi and A. S. Warsy, "Hypertension in Saudi Arabia," Saudi Journal of Kidney Diseases and Transplantation, Vol. 10, 1999, pp. 365-371.

[9] K. Nazim Uddin, "Prevalence of Hypertension in Saudi Arabia," Practitioner East Mediterranean Edition, Vol. 18, No. 4, 1994, pp. 805-806.

[10] M. M. Al-Nozha and A. K. Osman, "The Prevalence of Hypertension in Different Geographical Regions of Saudi Arabia," Annals of Saudi Medicine, Vol. 18, No. 4, 1998, pp. 401-407.

[11] http://www.who.int/research/en/

[12] www.oracle.com/technology/documentation/datamining. html

[13] J. Echfeldt, C. M. Hawkins and M. Proschan, "Major Outcomes in High-Risk Hypertensive Patients Randomized to Angiotensin-Converting Enzyme Inhibitor or Calcium Channel Blocker vs Diuretic: The Antihypertensive and Lipid Lowering Treatment to Prevent Heart Attack Trial (ALLHAT)," Journal of the American Medical Association, Vol. 288, 2002, pp. 2981-2997. doi:10.1001/jama.288.23.2981 